\title{
Dynamic Zoning Selection for Handwritten Character Recognition
}

\author{
Luciane Y. Hirabara ${ }^{1}$, Simone B.K. Aires ${ }^{1}$, Cinthia O.A. Freitas ${ }^{1}$, \\ Alceu S. Britto Jr. ${ }^{1}$, and Robert Sabourin ${ }^{2}$ \\ ${ }^{1}$ PUCPR-Pontificia Universidade Católica do Paraná \\ ${ }^{2}$ ETS-Ecole de Technologie Supérieure \\ \{luciane, cinthia, alceu, simone\}@ppgia.pucpr.br, \\ robert.sabourin@etsmtl.ca
}

\begin{abstract}
This paper presents a two-level based character recognition method in which a dynamically selection of the most promising zoning scheme for feature extraction allows us to obtain interesting results for character recognition. The first level consists of a conventional neural network and a look-up-table that is used to suggest the best zoning scheme for a given unknown character. The information provided by the first level drives the second level in the selection of the appropriate feature extraction method and the corresponding class-modular neural network. The experimental protocol has shown significant recognition rates for handwritten characters (from $80.82 \%$ to $88.13 \%)$.
\end{abstract}

Keywords: dynamic selection, zoning mechanism, handwritten character, recognition.

\section{Introduction}

An important subject of research in the field of document analysis and recognition is still the recognition of handwritten characters. The motivation is that even after many research efforts there is still a gap between human reading capabilities and the recognition systems. Over the years researchers have applied different techniques and methods to reduce this gap. In this direction, several authors have presented different schemes based on zoning mechanisms or regional decomposition methods. Zoning is a simple way to obtain local information and it has been used for extraction of topological information from patterns [1]. The goal of the zoning is to obtain local characteristics instead of global characteristics. This is possible, since a zoning scheme consists in partitioning the pattern bounding box in regions or zones. The resulting partitions allow us to determine the position of specific features of the pattern to be recognized [2]. However, the major problem related to zoning mechanisms is to choose the best zoning scheme to solve the recognition problem of different classes of characters.

Thus, depending on the domain of application or the experience of the researcher the zoning can be carried out exclusively on the basis of intuitive motivations [1] or based on the easier manner, i.e. fixed or symmetrical zoning [3-5]. Different zoning approaches for characters recognition can be found in the literature. By using 
IRONOFF handwritten database, the authors in [3] report recognition rates of $87.1 \%$ and $77.8 \%$ for uppercase and lowercase characters respectively, handling directly $2 \mathrm{D}$ pattern avoiding the subtle stage of the extraction of relevant and applying MLP-NN (Multiple Layer Perceptron - Neural Network). Other interesting results were reported in [5], where the recognition rate for lowercase characters was $80.75 \%$ and for uppercase characters was $89.21 \%$; applying Class-Modular MLP-NN and a feature set based on directional and curvature histograms for the contour image and a zoning mechanism into $Z=16(4 \times 4)$ or $Z=20$ (4 x 5) regions. Using the isolated French word images from IRONOFF database, the authors in [4] describes a system based on sliding window segmentation and 140 geometrical features are extracted from each frame. The classification stage is based on conventional discrete Hidden Markov Models (HMM) using Vector Quantization (VQ). This paper reported $83.1 \%$ of recognition rate for handwritten words.

The author in [6] uses a regular zoning scheme based on 4 symmetrical regions and reported $92.3 \%$ and $84.6 \%$ for upper and lowercase characters from NIST database. Using the same database, the work described in [7] uses an implicit zoning scheme based on row and columns discrete HMMs and reached $90.0 \%$, and $84.0 \%$ for upper and lowercase characters, respectively. Finally, the work presented in [8] describes an automatic approach to define the zoning for offline handwritten digit recognition, using Multi-Objective Evolutionary Algorithms (MOEAs). The authors pointed out that their proposal provides a self adaptive methodology to define the zoning strategy with $\mathrm{Z}$ non-overlapping zones and an acceptable error rate, with no need of human intervention during the search stage. The best result was obtained using six zones composed by three symmetrical rows (horizontal: 2/6, 2/6, 2/6) and three nonsymmetrical columns (vertical: $1 / 6,3 / 6,2 / 6$ ) using a random subset from the NIST SD-19 hsf-0123 handwritten digit database with 50,000 samples for the training set, and another 10,000 for the validation set to evaluate the individual's error rate. The error rate applying this zoning strategy was 5\%. However, the authors left an open problem: since, it is very difficult to find an unique and best zoning for all classes, is it possible to dynamically select the best zoning scheme for an unknown pattern?

To answer this question, this paper presents a dynamic zoning selection applied in a two-level method for character recognition. In the first level, a conventional Neural Network and a traditional zoning scheme based on four equal zones $(Z=4)$ is used to predict the top 3 recognition results for an unknown pattern. In fact, the idea is to predict the best zoning scheme from the different options shown in Figure 1. The second level uses the selected zoning to extract the features for a feedforward MLPNN using a Class-Modular architecture [9] that decides the final recognition result.

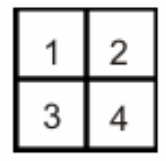

4

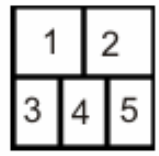

$5 \mathrm{H}$

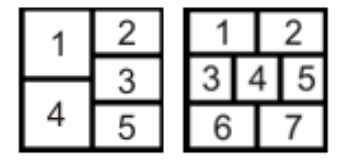

$5 \mathrm{~V}$

\section{7}

Fig. 1. Zoning Mechanisms: $\mathrm{Z}=4,5 \mathrm{H}, 5 \mathrm{~V}$ and 7 
The paper is organized as follows. Section 2 presents the baseline system and how the dynamic zoning selection is proposed. Section 3 presents the experimental results. Section 4 discusses the experimental results and points the future works.

\section{Baseline System}

The proposed method uses as input a 256 grey-level image. Then, a preprocessing step is applied, which is composed of binarization (OTSU algorithm [10]) and bounding box definition. The feature set is based on Concavities/Convexities deficiencies [11]. This feature set puts on evidence the topological and geometrical properties of the shape to be recognized and is computed by labeling the background pixels of the input images. The basic idea of concavity/convexity deficiencies is the following: for each background pixel in the image we search in four-directions: North, South, East, and West - Fig. 2. When black pixels are reached in all directions, we branch out in four auxiliary directions in order to confirm if the current white pixel is really inside a closed contour. Figure 2 shows the obtained result after the labeling process. The entire and definitive alphabet has 24 different symbols $(S=24)$ [2].

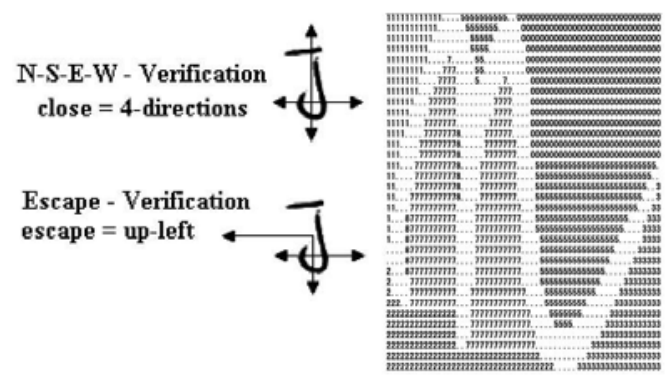

Fig. 2. Feature extraction: character " $n$ "

These global features are extracted considering the following zoning strategies: $Z=$ $4,5 \mathrm{H}, 5 \mathrm{~V}$ and 7 regions, as presented and defined by [2]. This hybrid feature extraction provided to the system a global and local description of the shape to be recognized.

The proposed classification method is based on two levels; (a) dynamic zoning selection (DZS) and (b) character recognition (CR), as depicted in Figure 3. The first level is composed of a conventional Neural Network classifier. Given an unknown character, the feature extraction is done using a classical zoning $(Z=4)$. The main objective of the first level is to provide the best zoning scheme for the second level. So, for this purpose, we previously calculate the confusion matrices of the class-modular Neural Networks trained based on the different zoning options: $Z=4,5 \mathrm{H}, 5 \mathrm{~V}$ and 7 regions as presented in Figure 1. Thus, the best zoning scheme is selected by looking for the confusion matrix that present the smaller number of confusions among the corresponding character classes involved in the Top 3 recognition result. The zoning scheme corresponding to the found matrix is selected for the feature extraction of the second level. However, in case of a tie when comparing the number of confusions, the best zoning scheme is chosen by considering only the Top 1 recognition result. In this 
case, the confusion matrix that presents the best recognition result for the Top 1 result is selected for feature extraction of the next level.

The second level of proposed method is based on a feedforward MLP-NN using Class-Modular architecture [9]. In Class-Modular architecture a single task is decomposed into multiple subtasks and each subtask is allocated to an expert network. In this paper, as well as in [9] the $K$-classification problem is decomposed into $K 2$ classication subproblems, one for each of the $K$ classes. A 2-classification subproblem is solved by the 2-classifier specifically designed for the corresponding class. The 2classifier is only responsible for one specific class and discriminates that class from the other $K$-1 classes. In the class-modular framework, $K 2$-classifiers solve the original $K$ classification problem cooperatively, and the class decision module integrates the outputs from the $K$ 2-classifiers.
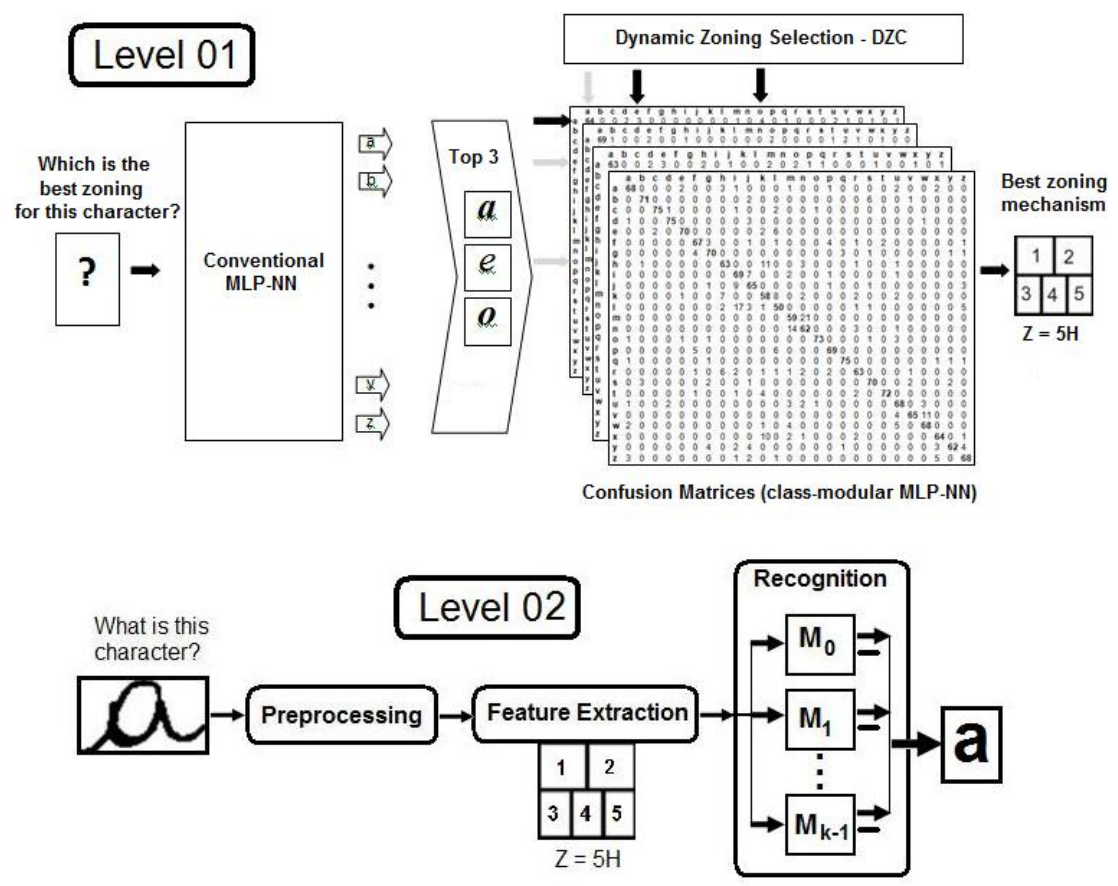

Fig. 3. Baseline system overview: Level 01(Dynamic Zoning Selection - DZS) and Level 02 (Character Recognition - CR)

At this level, the feature extraction is dynamic and depends on the zoning scheme selected in the first level.

\section{Experimental Results}

This section presents the main results undertaken during the development of the proposed method. The description of the used database and the results of the first and second levels of the proposed method are described. 


\subsection{Database}

The experiments were carried out using the lowercase handwritten character database from IRESTE/University of Nantes (France), called IRONOFF (IRESTE ON/OFF Dual Database), which is composed of isolated digits, isolated lowercase and uppercase characters and, isolated words from a 197 word lexicon. The IRONOFF database was selected because it is fully cursive. It was collected from about 700 writers, mainly of French nationality. The experiments were carried out using three subsets, nominated as the training, validation and testing sets. Their compositions are as follows: $60 \%, 20 \%$ and $20 \%$ for training, validation, and testing, respectively. The database sums up 10,400 images of lowercase handwritten characters [12].

\subsection{Conventional MLP-NN}

As described before, the first level of the proposed method consists of a conventional neural network classifier, which is composed of $N=96(24 \times Z$, where $Z=4)$ nodes in the input layer, a 59 number of nodes in the hidden layer, and an output layer with 26 nodes. Validation sets were employed in order to avoid overtraining and to make possible a Cross-Validation process. All the classes were trained together.

The proposed method considers the three major outputs (Top 3) for each sample of character class in the test database. We have experimentally evaluated different numbers of neurons in the hidden layer and the best results were achieved with 59 neurons. Firstly, the experiments consider the global feature extraction, without applying the zoning mechanism (Z-Global). Then, the classifier was experimented with the zoning schemes presented in Figure 1. We observed in Table 1 that there is an increase recognition rate when applying the $Z=4$ as strategy of zoning, as well as an increase when compared from $Z=4$ to $Z=7$. At this point is possible to define the best zoning mechanism for each class of character. This information is used to construct a kind of a look-up-table used in the first level of our method. In fact, the look-up-table consists in the confusion matrices derived from the use of the different zoning strategies and the class-modular MLP-NN. Thus, by analyzing the confusion among the three major outputs (TOP3), it is possible to define the more appropriated strategy of zoning. Thereby the zoning that had the small number of confusion among the Top 3 outputs is assigned to be used in the second level of the proposed method. As explained before, in case of a tie the zoning scheme of the top 1 recognition result is selected.

\subsection{Class Modular MLP-NN}

The second level of the proposed method is based on a Class-Modular MLP-NN, where each of $K$ 2-classifiers is trained independently of the other classes using the training and validation set. To train 2-classifiers for each character class $(K=26)$, we re-organize the samples in the original training and validation set into $K$-two groups, $\mathrm{Z} 0$ and $\mathrm{Z} 1$, such that $\mathrm{Z} 0$ contains the samples from the current class and $\mathrm{Z1}$ all the others, taking into account the a priori probability for each class. To recognize the input character patterns, the class decision module takes only the values of $O_{0}$ and uses the simple winner-takes-all scheme to determine the final class. Table 1 presents 
the recognition rate obtained for each zoning mechanism. All these results confirm that the class-modular network is superior in terms of convergence over the conventional network (according to the monitoring of the MSE-mean-square error) and in terms of recognition capability than the conventional network, as presented by [5] [9].

Table 1. Conventional NN and Class-Modular MLP-NN

\begin{tabular}{|c|c|c|c|c|c|c|c|c|c|}
\hline \multirow[t]{2}{*}{ Characters } & \multicolumn{5}{|c|}{$\begin{array}{c}\text { Conventional NN } \\
\text { Recognition Rate (\%) }\end{array}$} & \multicolumn{4}{|c|}{$\begin{array}{c}\text { Class-Modular MLP-NN } \\
\text { Recognition Rate (\%) }\end{array}$} \\
\hline & ZGlobal & $\mathbf{Z 4}$ & Z5H & $\mathrm{Z5V}$ & Z7 & $\mathbf{Z 4}$ & Z5H & $\mathrm{Z5V}$ & Z7 \\
\hline $\mathbf{a}$ & 68.75 & 83.75 & 82.50 & 80.00 & 83.75 & 80.00 & 86.25 & 78.75 & 85.00 \\
\hline b & 61.25 & 82.50 & 86.25 & 83.75 & 86.25 & 88.75 & 88.75 & 90.00 & 88.75 \\
\hline c & 71.25 & 95.00 & 97.50 & 93.75 & 95.00 & 93.75 & 95.00 & 88.75 & 93.75 \\
\hline d & 42.50 & 92.50 & 90.00 & 91.25 & 86.25 & 93.75 & 91.25 & 81.25 & 93.75 \\
\hline e & 76.25 & 83.75 & 83.75 & 77.50 & 63.75 & 82.50 & 83.75 & 77.50 & 87.50 \\
\hline f & 26.25 & 38.75 & 67.50 & 67.50 & 65.00 & 80.00 & 68.75 & 80.00 & 83.75 \\
\hline $\mathbf{g}$ & 40.00 & 65.00 & 71.25 & 67.50 & 61.25 & 63.75 & 65.00 & 73.75 & 87.50 \\
\hline h & 37.50 & 76.25 & 72.50 & 67.50 & 68.75 & 76.25 & 80.00 & 70.00 & 78.75 \\
\hline i & 80.00 & 81.25 & 82.50 & 82.50 & 77.50 & 86.25 & 88.75 & 86.25 & 86.25 \\
\hline $\mathbf{j}$ & 70.00 & 77.50 & 78.75 & 73.75 & 81.25 & 81.25 & 77.50 & 81.25 & 81.25 \\
\hline $\mathbf{k}$ & 61.25 & 66.25 & 73.75 & 62.50 & 76.25 & 65.00 & 72.50 & 72.50 & 72.50 \\
\hline 1 & 53.75 & 70.00 & 58.75 & 66.25 & 68.75 & 70.00 & 62.50 & 72.50 & 62.50 \\
\hline m & 13.75 & 48.75 & 71.25 & 60.00 & 61.25 & 50.00 & 53.75 & 85.00 & 73.75 \\
\hline $\mathrm{n}$ & 82.50 & 81.25 & 65.00 & 63.75 & 77.50 & 76.25 & 65.00 & 82.50 & 77.50 \\
\hline o & 82.50 & 88.75 & 90.00 & 93.75 & 91.25 & 88.75 & 87.50 & 90.00 & 91.25 \\
\hline p & 76.25 & 81.25 & 87.50 & 82.50 & 87.50 & 76.25 & 87.50 & 88.75 & 86.25 \\
\hline q & 87.50 & 95.00 & 93.75 & 98.75 & 97.50 & 95.00 & 92.50 & 88.75 & 93.75 \\
\hline $\mathbf{r}$ & 65.00 & 71.25 & 67.50 & 72.50 & 73.75 & 77.50 & 72.50 & 66.25 & 78.75 \\
\hline $\mathbf{s}$ & 76.25 & 85.00 & 77.50 & 81.25 & 86.25 & 88.75 & 78.75 & 81.25 & 87.50 \\
\hline t & 86.25 & 91.25 & 78.75 & 91.25 & 87.50 & 87.50 & 88.75 & 87.50 & 90.00 \\
\hline $\mathbf{u}$ & 50.00 & 83.75 & 83.75 & 78.75 & 82.50 & 87.50 & 83.75 & 68.75 & 85.00 \\
\hline $\mathbf{v}$ & 62.50 & 71.25 & 81.25 & 83.75 & 83.75 & 80.00 & 82.50 & 80.00 & 81.25 \\
\hline $\mathbf{w}$ & 83.75 & 88.75 & 92.50 & 87.50 & 90.00 & 83.75 & 87.50 & 90.00 & 85.00 \\
\hline $\mathbf{x}$ & 82.50 & 88.75 & 82.50 & 86.25 & 86.25 & 83.75 & 86.25 & 85.00 & 80.00 \\
\hline y & 90.00 & 92.50 & 90.00 & 92.50 & 87.50 & 90.00 & 90.00 & 87.50 & 77.50 \\
\hline $\mathbf{z}$ & 88.75 & 87.50 & 86.25 & 88.75 & 95.00 & 82.50 & 85.00 & 88.75 & 85.00 \\
\hline & 66.01 & 79.52 & 80.48 & 79.81 & 80.82 & 81.11 & 80.82 & 81.63 & 83.61 \\
\hline
\end{tabular}

\subsection{Dynamic Zoning Selection}

The conventional Neural Network classifier in the first level provides the top 3 recognition result and the most promising zoning scheme based on the confusion matrices previously created by considering different zoning schemes. The second level uses such information for feature extraction and selection of the appropriated Class-Modular MLP-NN. By applying the dynamic zoning selection - DZC (first level based on class-modular MLP-NN) and character recognition - CR (second level based on class-modular MLP-NN) the system reached $88.13 \%$ of average rate. Table 2 presents the recognition rates for each class of handwritten characters. 


\subsection{Related Works}

Table 3 presents the results of some related works that uses the IRONOFF database. Comparing results is not easy since the works refers to different sets, feature extraction and classification strategies. Taking into consideration these differences, the results indicate that the dynamic zoning selection (DZS) is promising and the recognition rates are comparable to the literature.

Table 2. DZS (Level 01) + Character Recognition (Level 02)

\begin{tabular}{c|c|c|c}
\hline Character & Rec. Rate (\%) & Character & Rec. Rate (\%) \\
\hline $\mathbf{a}$ & 85.00 & $\mathbf{0}$ & 92.50 \\
\hline $\mathbf{b}$ & 91.25 & $\mathbf{~}$ & 92.50 \\
\hline $\mathbf{c}$ & 96.25 & $\mathbf{q}$ & 95.00 \\
\hline $\mathbf{d}$ & 93.75 & $\mathbf{~}$ & 82.50 \\
\hline $\mathbf{e}$ & 87.50 & $\mathbf{s}$ & 95.00 \\
\hline $\mathbf{f}$ & 86.25 & $\mathbf{t}$ & 92.50 \\
\hline $\mathbf{g}$ & 88.75 & $\mathbf{u}$ & 91.25 \\
\hline $\mathbf{h}$ & 83.75 & $\mathbf{~}$ & 86.25 \\
\hline $\mathbf{i}$ & 90.00 & $\mathbf{w}$ & 90.00 \\
\hline $\mathbf{j}$ & 78.75 & $\mathbf{x}$ & 87.50 \\
\hline $\mathbf{k}$ & 80.00 & $\mathbf{y}$ & 91.25 \\
\hline $\mathbf{l}$ & 80.00 & $\mathbf{z}$ & 88.75 \\
\hline $\mathbf{m}$ & 82.50 & Average & $\mathbf{8 8 . 1 3}$ \\
\hline $\mathbf{n}$ & 82.50 & \multicolumn{3}{|l}{}
\end{tabular}

Table 3. Related works (IRONOFF databases)

\begin{tabular}{l|c|c|c|c}
\hline \multicolumn{1}{c|}{ Method } & Tr & V & Ts & Rec. (\%) \\
\hline [4] - IRONOFF - words & & & & 83.1 \\
\hline [3] - IRONOFF & 7,953 & -- & 3,926 & 87.1 \\
uppercase & 7,952 & -- & 3,916 & 77.8 \\
lowercase & 6,240 & 2,080 & 2,080 & 83.0 \\
\hline [2] - IRONOFF & 6,240 & 2,080 & 2,080 & 84.7 \\
uppercase $(Z=4)$ & -- & -- & -- & 80.7 \\
\hline uppercase $(Z=7)$ & 6,240 & 2,080 & 2,080 & 88.1 \\
\hline [16] - IRONOFF - lowercase & \multicolumn{1}{|c|}{} \\
\hline Proposed Method - lowercase & & &
\end{tabular}

\section{Conclusion and Future Works}

This paper presented a two-level based character recognition method in which a dynamically zoning selection (DZS) scheme for feature extraction allows us to obtain promising results for character recognition. In the first level a conventional MLP-NN and the analysis of confusion matrices are used to determine the most promising zoning scheme for a given unknown character. The information provided by the first level drives the second level in the selection of the appropriate feature extraction 
method and the corresponding class-modular neural network. The experimental results have shown some significant improvement in the recognition rates for lowercase handwritten characters from $80.82 \%$ (Conventional MLP-NN $-\mathrm{Z}=7$ ) to $88.13 \%(\mathrm{DZS}+\mathrm{CR})$.

\section{References}

1. Lecce, V., Dimauro, G., Guerriero, A., Impedovo, S., Pirlo, G., Salzo, A.: Zoning design for handwritten numerical recogniotion. In: 7th Int. Workshop on Frontiers in Handwriting Recognition, pp. 583-588 (2000)

2. Freitas, C.O.A., Oliveira, L.E.S., Bortolozzi, F., Aires, S.B.K.: Handwritten Character Recognition using Non-Symmetrical Percpetual Zoning. International Journal of Pattern Recognition and Artificial Intelligence (IJPRAI) 21(1), 1-21 (2007)

3. Poisson, E., Viard-Gaudin, C., Lallican, P.M.: Multi-Modular architecture based on convolutional Neural networks for online handwritten character recognition. In: International Conference on Neural Information Processing, vol. 5, pp. 2444-2448 (2002)

4. Tay, Y.H., Lallican, P.M, Khalid, M., Gaudin, C.V, Knerr, S.: An Offline Cursive Handwritten Word Recognition System. In: IEEE Region 10 Conference, TENCON, Singapore, pp. 19-22 (2001)

5. de Avila, S., Matos, L., Freitas, C., de Carvalho, J.M.: Evaluating a Zoning Mechanism and Class-Modular Architecture for Handwritten Characters Recognition. In: Rueda, L., Mery, D., Kittler, J. (eds.) CIARP 2007. LNCS, vol. 4756, pp. 515-524. Springer, Heidelberg (2007)

6. Koerich, A.L.: Large Vocabulary Off-Line Handwritten Word Recognition, PhD thesis, École de Technologie Supérieure, Montreal-Canada (August 2002)

7. Britto Jr., A.S., Sabourin, R., Bortolozzi, F., Suen, C.Y.: Foreground and background information in an HMM-Based method for recognition of isolated characters and numeral strings. In: 9th Int. Workshop on Frontiers in Handwriting Recognition (IWFHR-9), Tokio Japan, pp. 371-376 (2004)

8. Radtke, P.V.W., Oliveira, L.S., Sabourin, R., Wong, T.: Intelligent zoning design using multi-objective evolutionary algorithms. In: 7th Int. Conf. Document Analysis and Recognition ICDAR, pp. 824-828 (2003)

9. Oh, I.-S., Suen, C.Y.: A class-modular feedforward neural network for handwriting recognition. Pattern Recognition 35, 229-244 (2002)

10. Otsu, N.: A threshold selection method from gray-level histograms. IEEE Trans. Syst. Man. Cybern. 9(1), 63-66 (1979)

11. Parker, J.R.: Algorithms for Image Processing and Computer Vision. John Wiley (1997)

12. Viard-Gaudin, C.: The ironoff user manual, IRESTE, University of Nantes, France (1999) 